\title{
Activation of TRPV1-dependent calcium oscillation exacerbates seawater inhalation-induced acute lung injury
}

\author{
CONGCONG LI ${ }^{1 *}$, LIYAN BO ${ }^{1 *}$, QINGQING LIU ${ }^{1}$, WEI LIU ${ }^{1}$, \\ XIANGJUN CHEN ${ }^{1}$, DUNQUAN XU ${ }^{2}$ and FAGUANG JIN ${ }^{1}$ \\ ${ }^{1}$ Department of Respiration, Tangdu Hospital, Fourth Military Medical University, Xi'an, Shaanxi 710038; \\ ${ }^{2}$ Department of Pathology and Pathophysiology, Fourth Military Medical University, Xi'an, Shaanxi 710032, P.R. China
}

Received April 27, 2015; Accepted December 21, 2015

DOI: $10.3892 / \mathrm{mmr} .2016 .4804$

\begin{abstract}
Calcium is an important second messenger and it is widely recognized that acute lung injury (ALI) is often caused by oscillations of cytosolic free $\mathrm{Ca}^{2+}$. Previous studies have indicated that the activation of transient receptor potential-vanilloid (TRPV) channels and subsequent $\mathrm{Ca}^{2+}$ entry initiates an acute calcium-dependent permeability increase during ALI. However, whether seawater exposure induces such an effect through the activation of TRPV channels remains unknown. In the current study, the effect of calcium, a component of seawater, on the inflammatory reactions that occur during seawater drowning-induced ALI, was examined. The results demonstrated that a high concentration of calcium ions in seawater increased lung tissue myeloperoxidase activity and the secretion of inflammatory mediators, such as tumor necrosis factor- $\alpha$ (TNF- $\alpha$ ) and interleukin (IL)- $1 \beta$ and IL-6. Further study demonstrated that the seawater challenge elevated cytosolic $\mathrm{Ca}^{2+}$ concentration, indicated by $\left[\mathrm{Ca}^{2+}\right] \mathrm{c}$, by inducing calcium influx from the extracellular medium via TRPV1 channels. The elevated $\left[\mathrm{Ca}^{2+} \mathrm{c}\right]$ may have resulted in the increased release of TNF- $\alpha$ and IL- $1 \beta$ via increased phosphorylation of nuclear factor $-\kappa \mathrm{B}(\mathrm{NF}-\kappa \mathrm{B})$. It was concluded that a high concentration of calcium in seawater exacerbated lung injury, and TRPV1 channels were notable mediators of the calcium increase initiated by the seawater challenge. Calcium influx through TRPV1 may have led to greater phosphorylation of NF- $\mathrm{B}$ and increased release of TNF- $\alpha$ and IL-1 $\beta$.
\end{abstract}

Correspondence to: Professor Faguang Jin, Department of Respiration, Tangdu Hospital, Fourth Military Medical University, 1 Xinsi Road, Xi'an, Shaanxi 710038, P.R. China

E-mail: jinfag@fmmu.edu.cn

${ }^{*}$ Contributed equally

Key words: transient receptor potential-vanilloid 1, lung injury, calcium oscillation, inflammation

\section{Introduction}

Drowning is a major, but often neglected, public health problem (1). Drowning is the third leading cause of accidental mortality, with $>388,000$ mortalities per year worldwide (2). Water inhalation can induce acute lung injury (ALI) and acute respiratory distress syndrome by disturbing the barrier function of alveolar epithelium, leading to lung edema and inflammatory reactions (3-5). Previous research has demonstrated that intracellular calcium $\left(\mathrm{Ca}^{2+}\right)$ oscillations are vital in ALI, as they lead to reduced integrity of the blood-air barrier $(6,7)$, increased NF- $\kappa \mathrm{B}$ activation and lung inflammation (7). It was also indicated that intracellular $\mathrm{Ca}^{2+}$ oscillations are dependent on extracellular $\mathrm{Ca}^{2+}(7)$, and an increased infiltration coefficient can be prevented in low- $\mathrm{Ca}^{2+}$ lung perfusate (8). Therefore, modulating calcium signaling may provide beneficial effects in cases of ALI induced by seawater aspiration.

Transient receptor potential-vanilloid (TRPV) is a family of plasma membrane ion channels consisting of seven members (TRPV1-7) (9). They are a notable receptor family that respond to a wide variety of endogenous and exogenous stimuli, including temperature $(10,11)$, proinflammatory mediators $(11-13), \mathrm{pH}(11,14)$, stretch $(15)$ and osmolality $(11,16,17)$. Two members of the TRPV family (TRPV1 and 4) have been identified to participate in ALI $(15,18)$. As a cell membrane-bound $\mathrm{Ca}^{2+}$ channel, activation of TRPV1 is an important factor in intracellular $\mathrm{Ca}^{2+}$ oscillations following exposure to cytokines, abnormal $\mathrm{pH}$ and osmolality (12,18-20). TRPV4 is also a $\mathrm{Ca}^{2+}$-permeable cation channel gated by a variety of external factors (17), including heat (21), membrane stretch, osmotic changes (22) and mechanical stimuli (23). Previous studies have indicated that the activation of TRPV4 and subsequent $\mathrm{Ca}^{2+}$ entry initiated an acute calcium-dependent permeability increase following lung injury resulting from 14,15-epoxyeicosatrienoic acid (14,15-EET), 5,6-EET and 8,9-EET (6) exposure, in addition to ventilator-induced lung injury (15). These results imply that TRPV is important during ALI and is a potential therapeutic target for the treatment of lung injury. Whether seawater exposure induces ALI through the activation of TRPV remains unknown. The role of the interaction between hypertonia and high calcium concentration in seawater instillation-induced ALI required further investigation. 
In the present study, the hypothesis that seawater instillation induces ALI through the activation of TRPV and subsequent intracellular calcium oscillations was analyzed and the interaction between hypertonia and high calcium concentration during ALI was examined.

\section{Materials and methods}

Reagents. The following reagents were used in the current study: Monoclonal mouse $\beta$-actin (1:8,000 dilution; cat. no. A5441; Sigma-Aldrich, St. Louis, MO, USA); monoclonal rabbit phospho-NF-кB p65 (Ser536; 1:500 dilution; cat. no. 3033; Cell Signaling Technology, Inc., Danvers, MA, USA) and monoclonal rabbit NF-кB p65 (1:500 dilution; cat. no. 8242; Cell Signaling Technology, Inc.) antibodies. Acti-stain 488 phalloidin (Cytoskeleton, Inc., Denver, USA); enzyme-linked immunosorbent assay (ELISA) kits (R\&D Systems, Inc., Minneapolis, MN, USA); myeloperoxidase (MPO) activity assay kit (Nanjing Jiancheng Bioengineering Institute, Nanjing, China); Fluo-3,AM and Pluronic F-127 (Biotium, Inc., Hayward, CA, USA); ruthenium red, capsazepine and BAPTA-AM (Abcam); HC067047 and EGTA (Sigma-Aldrich). Seawater was prepared according to the major compositions of the East China Sea provided by the Chinese Ocean Bureau (osmolality, 1,300 mmol/l; pH 8.2; NaCl, $26.518 \mathrm{~g} / \mathrm{l} ; \mathrm{MgSO}_{4}$, $3.305 \mathrm{~g} / \mathrm{l} ; \mathrm{MgCl}_{2}, 2.447 \mathrm{~g} / \mathrm{l} ; \mathrm{CaCl}_{2}, 1.141 \mathrm{~g} / \mathrm{l} ; \mathrm{KCl}, 0.725 \mathrm{~g} / \mathrm{l}$; $\left.\mathrm{NaHCO}_{3}, 0.202 \mathrm{~g} / \mathrm{l} ; \mathrm{NaBr}, 0.083 \mathrm{~g} / \mathrm{l}\right)$.

Cell culture and treatment. The epithelial cell line A549 (American Type Culture Collection, Rockville, MD, USA), derived from lung adenocarcinoma was cultured in RPMI 1640 medium (HyClone Laboratories, Inc., Logan, UT, USA) supplemented with $10 \%$ fetal bovine serum (Zhejiang Tianhang Biotechnology Co., Ltd., Huzhou, China) at $37^{\circ} \mathrm{C}$ and in a $5 \% \mathrm{CO}_{2}$ atmosphere. The cells were treated with seawater by addition to the culture medium at a $25 \%$ volume ratio. The cells and supernatant were harvested $4 \mathrm{~h}$ after exposure to seawater.

Single cell $\left[\mathrm{Ca}^{2+}\right] \mathrm{c}$ measurement. A549 cells grown in culture were exposed to culture medium containing the $\mathrm{Ca}^{2+}$-sensitive fluorescent indicator, Fluo-3,AM $(5 \mu \mathrm{M})$, and a nonionic dispersing agent, Pluronic F-127 (0.025\%), for $30 \mathrm{~min}$ at $37^{\circ} \mathrm{C}$. Following the loading period, the medium was replaced, and the cells were incubated for a further $30 \mathrm{~min}$. Fluorescence intensity, reflecting the concentration of $\left[\mathrm{Ca}^{2+}\right] \mathrm{c}$, was recorded by confocal laser-scanning microscopy (FV10; Olympus, Tokyo, Japan). The re-addition of calcium was performed by adding $\mathrm{CaCl}_{2}$. The liquid above the cells was replaced with the seawater with a normal concentration of $\mathrm{CaCl}_{2}(1.141 \mathrm{~g} / \mathrm{l})$. Images were captured for quantification. The groups and their treatments were as follows: Control group: no extra treatment with the exception of loading the Fluo-3; seawater group: Following loading with Fluo-3 AM $(5 \mu \mathrm{M})$, the cells were exposed to seawater challenge at the predetermined time; BAPTA-AM group: Cells were loaded with Flou-3 AM $(5 \mu \mathrm{M})$ and BAPTA-AM $(5 \mu \mathrm{M}) 30 \mathrm{~min}$ prior to exposure to seawater challenge at the predetermined time; EGTA group: Following loading with Fluo-3 AM $(5 \mu \mathrm{M})$, the cells were exposed to $\mathrm{Ca}^{2+}$-free seawater with $1 \mathrm{mM}$ EGTA at the predetermined time; ruthenium red group: Cells were loaded with Flou-3 AM $(5 \mu \mathrm{M})$ and ruthenium red $(3 \mu \mathrm{M}) 30 \mathrm{~min}$ prior to exposure to seawater challenge at the predetermined time; HCO67047 group: Cells were loaded with Flou-3 AM $(5 \mu \mathrm{M})$ and HCO67047 $(1 \mu \mathrm{M}) 30 \mathrm{~min}$ prior to exposure to seawater challenge at the predetermined time; capsazepine group: Cells were loaded with Flou-3 AM $(5 \mu \mathrm{M})$ and capsazepine $(10 \mu \mathrm{M}) 30 \mathrm{~min}$ prior to exposure to seawater challenge at the predetermined time.

ELISA assay. Levels of TNF- $\alpha$, IL-1 $\beta$ and IL- 6 in lung tissues were determined using the ELISA kits. Lung tissues were homogenized in cool phosphate-buffered saline (PBS) at a 1:5 ratio of lung tissue to PBS. Assays were conducted according to the manufacturer's instructions. The groups and their treatments were as follows: Control group: Cells were treated with $25 \%$ PBS and 75\% RPMI-1640 for 4 h; seawater group: Cells were exposed to $25 \%$ seawater and $75 \%$ RPMI-1640 for $4 \mathrm{~h}$; BAPTA-AM group: Cells were loaded with BAPTA-AM (5 $\mu \mathrm{M})$ for $30 \mathrm{~min}$ and then exposed to $25 \%$ seawater and $75 \%$ RPMI-1640 for 4 h; EGTA group: Cells were exposed to $25 \%$ $\mathrm{Ca}^{2+}$-free seawater with $1 \mathrm{mM}$ EGTA and 75\% RPMI-1640 for $4 \mathrm{~h}$; seawater + PDTC group: Cells were loaded with PDTC $(200 \mu \mathrm{M})$ for $30 \mathrm{~min}$ and then exposed to $25 \%$ seawater and 75\% RPMI-1640 for $4 \mathrm{~h}$; seawater + HCO67047 group: Cells were loaded with HCO67047 $(1 \mu \mathrm{M})$ for $30 \mathrm{~min}$ and then exposed to $25 \%$ seawater and $75 \%$ RPMI-1640 for $4 \mathrm{~h}$; seawater + capsazepine group: Cells were loaded with capsazepine $(10 \mu \mathrm{M})$ for $30 \mathrm{~min}$ and then exposed to $25 \%$ seawater and $75 \%$ RPMI-1640 for $4 \mathrm{~h}$.

Animal procedures. Adult male Sprague-Dawley (SD) rats weighing 180-200 g were purchased from the Laboratory Animal Centre of the Fourth Military Medical University (Xi'an, China) and housed under a light/dark cycle of 12/12 h, with standard food and water provided ad libitum. All procedures used in the present study were approved by the Animal Care and Use Committee of the Fourth Military Medical University. Rats were anesthetized with $1.5 \%$ sodium pentobarbital (50 mg/kg; Sigma-Aldrich) followed by intratracheal administration of seawater ( $4 \mathrm{ml} / \mathrm{kg}$ body weight) into the lungs within 4 min via a 20 -gauge intravenous catheter through the trachea. The rats were maintained in a supine position with a $30^{\circ}$ head-up tilt during the experiments. The rats were euthanized with a sodium pentobarbital overdose $(500 \mathrm{mg} / \mathrm{kg}$ ) at the predetermined points of time and then the lungs were harvested for further experiments. SD rats were randomly assigned into the following four groups $(n=4)$ : Control group: Rats with no intervention; seawater group: Rats were intratracheally administered seawater $(4 \mathrm{ml} / \mathrm{kg}$ body weight) into the lungs within 4 min via a 20 -gauge intravenous catheter through the trachea; $\mathrm{Ca}^{2+}$-free seawater group: Rats were intratracheally administered $\mathrm{Ca}^{2+}$-free seawater $(4 \mathrm{ml} / \mathrm{kg}$ body weight) into the lungs; isotonic seawater group: Rats were intratracheally administered isotonic seawater with no change in calcium concentration.

Western analysis. Protein was extracted from the lower right lung by homogenization and centrifugation at 10,000 x $\mathrm{g}$ for $20 \mathrm{~min}$ at $4^{\circ} \mathrm{C}$. Proteins were separated by $10 \%$ SDS-PAGE 
(120 v; Nanjing Jiancheng Bioengineering Institute, Nanjing, China) and were transferred onto a nitrocellulose membrane (Pall Corp., Washington, NY, USA). The membranes were blocked with 5\% non-fat dry milk in Tris-buffered saline (TBS) and probed with the antibodies against phospho-NF- $\mathrm{B}$ p65 (Ser536; dilution, 1:500), NF- $\mathrm{KB}$ p65 (dilution, 1:500) and $\beta$-actin (dilution, 1:8,000). Following incubation with the primary antibody overnight, the membranes were washed with TBS-Tween 20 and incubated with horseradish peroxidase-conjugated secondary antibody (dilution, 1:10,000). Target proteins were detected by the enhanced chemiluminescence detection system (WesternBright ECL-spray ; Advansta, Inc., Menlo Park, CA, USA). Four samples from each group were used for densitometry analysis (version 4.6.2; Quantity One software; Bio-Rad Laboratories, Inc., Hercules, CA, USA).

Confocal visualization of F-actin. A549 cells were cultured on coverslips and exposed to the different treatment conditions, including the control (no treatment), seawater-treated, seawater + BAPTA-AM and sewater + capsazepine groups. Following treatments, cells were fixed and permeabilized at room temperature, washed with PBS and incubated with $100 \mathrm{nM}$ Acti-stain 488 phalloidin in the dark for $30 \mathrm{~min}$. The nuclei were visualized by DAPI (4',6-diamidino-2-phenylindole; Beyotime Institute of Biotechnology, Shanghai, China) staining This stain was excited using a $340 \mathrm{~nm}$ laser and detected by confocal laser-scanning microscopy. The groups were treated as described above.

Histopathological evaluation. The lung tissues of the lower lobe of the right lung harvested from each rat were fixed with $4 \%$ paraformaldehyde (Sigma-Aldrich) for $24 \mathrm{~h}$ and embedded in paraffin and (Sigma-Aldrich) cut into 5- $\mu \mathrm{m}$ sections. The sections were stained with hematoxylin and eosin (Sigma-Aldrich) prior to visualization at x200 magnification under a light microscope (CX41; Olympus).

MPO activity assay. The extent of neutrophil accumulation in the lung samples was measured by assaying MPO activity. Following homogenization and centrifugation $(10,000 \mathrm{x} \mathrm{g})$ of these lung tissues in cool PBS, MPO activity was determined by colorimetric analysis using a SmartSpec Plus spectrophotometer (Bio-Rad, Laboratories, Inc.), according to the manufacturer's instructions. The MPO activity was expressed as $\mathrm{U} / \mathrm{mg}$ protein.

Evans blue extravasation assessment. Barrier permeability of the lungs was measured by Evans blue extravasation analysis, and $30 \mathrm{~min}$ prior to instillation of seawater, Evans blue dye (Sigma-Aldrich; $20 \mathrm{mg} / \mathrm{kg}$ ) was injected into the rats through the tail vein. Normal saline was injected into the right ventricle immediately prior to euthanization. Once clear fluid was effused from the left atrium, the lower lobe of the right lung was removed. Evans blue dye was extracted from the tissue by incubation of the lung lobes in formamide (Sigma-Aldrich; $3 \mathrm{ml} / 100 \mathrm{mg})$ for $24 \mathrm{~h}$. Total Evans blue $(\mu \mathrm{g} / \mathrm{g})$ was calculated using spectrophotometry (620 nm; SmartSpec Plus; Bio-Rad, Laboratories, Inc.).
Bronchoalveolar lavage fluid (BALF) analysis. Following anesthetization of the rats, the lungs were lavaged with $1 \mathrm{ml}$ ice-cold PBS three times. The number of total cells and neutrophils in the BALF was calculated using Wright's staining (Sigma-Aldrich). The cells in the BALF were collected by centrifugation at $2,500 \mathrm{x}$ g, stained with Wright's stain according the manufacturer's instructions and then neutrophils that were dyed pale purple were counted using a cell counting plate.

Statistical analysis. All data are expressed as the mean \pm standard deviation. Statistically significant differences between the different groups were assessed using analysis of variance with a Bonferroni post-hoc test. $\mathrm{P}<0.05$ was considered to indicate a statistically significant difference.

\section{Results}

Seawater challenge elevated cytosolic $\left[\mathrm{Ca}^{2+}\right] \mathrm{c}$ by inducing calcium entry from extracellular medium. It was hypothesized that seawater inhalation leads to ALI by stimulating $\mathrm{Ca}^{2+}$ entry into the cytosol. In order to address this issue, a series of experiments were performed to examine the effects of seawater composed of modified components on the $\left[\mathrm{Ca}^{2+}\right] \mathrm{c}$ in A549 cells. The effects of seawater challenge on the $\left[\mathrm{Ca}^{2+}\right] \mathrm{c}$ were recorded using confocal laser-scanning microscopy to measure the fluorescent $\mathrm{Ca}^{2+}$-sensitive indicator, Fluo-3, AM, and images were captured for quantitative analysis. A rise in the fluorescence intensity indicated an increase in $\left[\mathrm{Ca}^{2+}\right] \mathrm{c}$. As presented in Fig. 1A and B, seawater exposure evoked a rapid $\left[\mathrm{Ca}^{2+}\right] \mathrm{c}$ increase and the increase reached a maximal value within $30 \mathrm{sec}$, followed by a trifling recovery and a sustained plateau. Subsequently, a parallel experiment in which cells were treated with $5 \mu \mathrm{M}$ BAPTA-AM (a selective intracellular $\mathrm{Ca}^{2+}$ chelator) for $40 \mathrm{~min}$ prior to seawater exposure demonstrated that pretreatment with a chelator completely abolished the elevation of $\left[\mathrm{Ca}^{2+}\right] \mathrm{c}$ induced by seawater (Fig. 1C).

Next, to clarify the source of $\mathrm{Ca}^{2+}$ ions, experiments were performed to evaluate whether seawater challenge elevated $\left[\mathrm{Ca}^{2+}\right] \mathrm{c}$ via release of $\mathrm{Ca}^{2+}$ from intracellular stores or influx of extracellular $\mathrm{Ca}^{2+}$. As presented in Fig. 1D, an extracellular $\mathrm{Ca}^{2+}$ chelator, EGTA, decreased the increase of $\left[\mathrm{Ca}^{2+}\right] \mathrm{c}$ induced by seawater. The inhibitory effect was cancelled by re-addition of $\mathrm{Ca}^{2+}$ (Fig. 1D). Thus, it was concluded that the elevation of $\left[\mathrm{Ca}^{2+}\right] \mathrm{c}$ evoked by seawater exposure was predominantly accomplished by increasing $\mathrm{Ca}^{2+}$ influx from extracellular sources. The effects of the different treatments on $\left[\mathrm{Ca}^{2+}\right] \mathrm{c}$ are summarized in Fig. 1E.

Calcium chelation by EGTA or BAPTA-AM reduced the release of inflammatory mediators following seawater exposure. To explore the consequences of $\left[\mathrm{Ca}^{2+}\right] \mathrm{c}$ elevation, the concentrations of the proinflammatory cytokines, TNF- $\alpha$ and IL-1 $\beta$, were measured in the supernatant. Following seawater exposure, the levels of TNF- $\alpha$ and IL-1 $\beta$ were significantly increased compared with the control group $(\mathrm{P}<0.001$ and $\mathrm{P}<0.01$, respectively; Fig. 2). These alterations were alleviated when cells were treated with BAPTA-AM or EGTA (Fig. 2).

Seawater challenge evoked extracellular $\mathrm{Ca}^{2+}$ influx by activating TRPVI channels. It was reported that TRPV4 initiates 
A

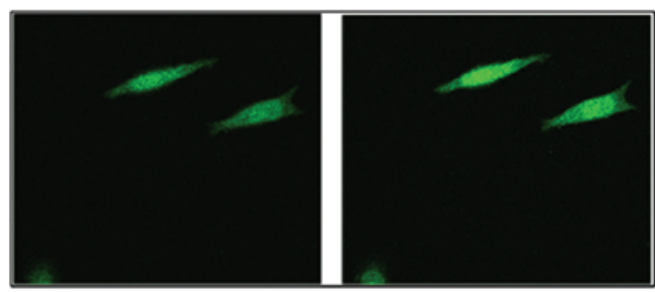

C

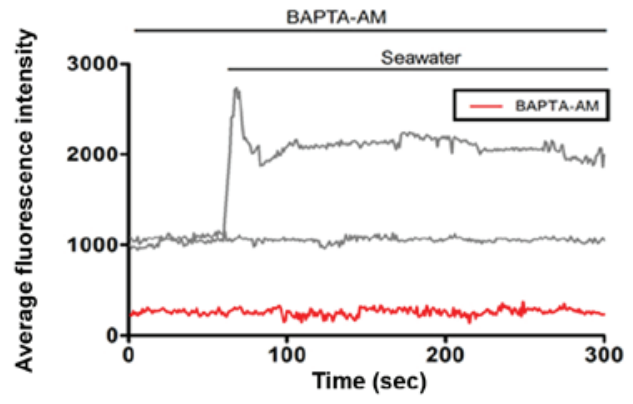

B

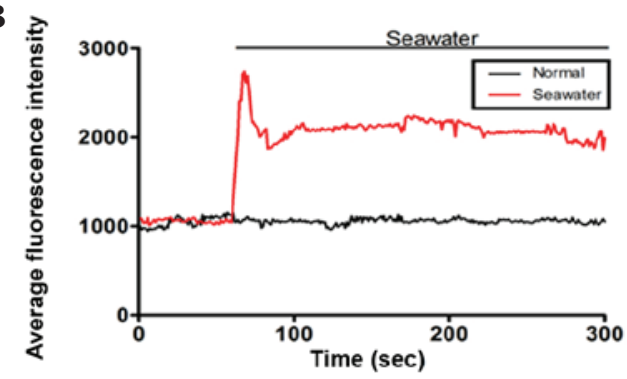

D

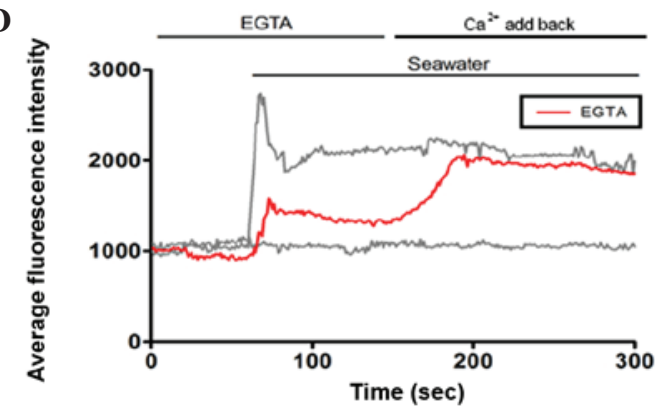

$\mathbf{E}$

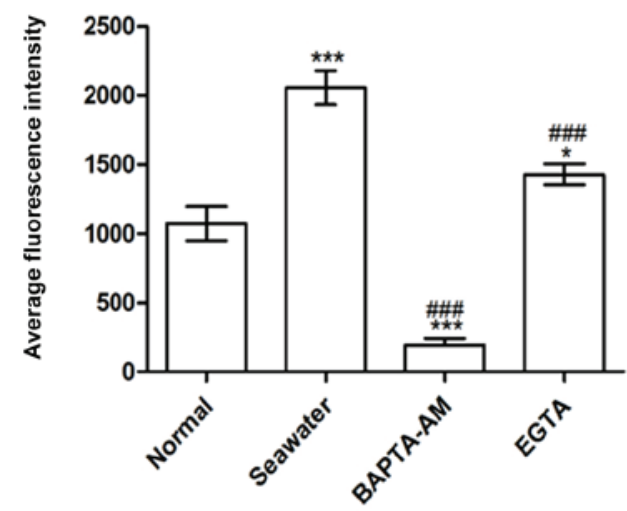

Figure 1. Effect of seawater exposure on the average fluorescence intensity in A549 cells. (A) Seawater exposure evoked a rapid fluorescence intensity increase that represented $\left[\mathrm{Ca}^{2+}\right] \mathrm{c}$ elevation. Left panel, A549 cells without stimulation; right panel, A549 cells exposed to seawater. (B) Quantification of fluorescence intensity following seawater treatment. (C) Pretreatment with BAPTA-AM may reverse the elevation of [Ca $\left.{ }^{2+}\right]$ c. (D) Chelation of calcium with EGTA reduced the rise of $\left[\mathrm{Ca}^{2+}\right] \mathrm{c}$ triggered by seawater. (E) Average fluorescence intensities for all groups. ${ }^{*} \mathrm{P}<0.05$ and ${ }^{* * * *} \mathrm{P}<0.001$ vs. the normal group; ${ }^{\# \# \# ~} \mathrm{P}<0.001$ vs. the seawater group.

the acute calcium-dependent permeability increase during ventilator-induced lung injury in isolated mouse lungs (6). The current study investigated whether similar membrane transport pathways were required for seawater exposure-induced extracellular $\mathrm{Ca}^{2+}$ influx. Subsequent experiments were then performed to identify the membrane transport pathway that mediated $\mathrm{Ca}^{2+}$ influx. Cells were treated with a range of inhibitors of potential $\mathrm{Ca}^{2+}$ entry channels $30 \mathrm{~min}$ prior to seawater exposure. The TRPV1-6 inhibitor ruthenium red significantly reduced the $\mathrm{Ca}^{2+}$ response to seawater challenge but did not abolish it (Fig. 3A). However, a potent selective TRPV4 antagonist, HC067047, had no observed effect on the seawater-induced increase of $\left[\mathrm{Ca}^{2+}\right] \mathrm{c}$ (Fig. 3B). Notably, the elevated level of the $\left[\mathrm{Ca}^{2+}\right] \mathrm{c}$ response to seawater exposure was reduced by treatment with the TRPV1-specific inhibitor, capsazepine (Fig. 3C). These results suggested that, unlike ventilator-induced lung injury, extracellular $\mathrm{Ca}^{2+}$ influx through TRPV1 channels was crucial to the increase of $\left[\mathrm{Ca}^{2+}\right] \mathrm{c}$ observed in A549 cells following exposure to seawater.
Seawater exposure induced $T N F-\alpha$ and $I L-1 \beta$ release through TRPVI activation and NF- $\kappa B$ phosphorylation. To establish the role of TRPV1 in mediating $\mathrm{Ca}^{2+}$ influx and the subsequent inflammatory reactions, the phosphorylation of NF- $\mathrm{KB}$ and the concentration of TNF- $\alpha$ and IL- $1 \beta$ in the supernatant were measured. Fig. 4 indicates that NF- $\mathrm{KB}$ phosphorylation was increased following seawater stimulation $(\mathrm{P}<0.001$, compared with the control group), whereas capsazepine abolished this effect $(\mathrm{P}<0.05$, compared with the seawater treatment).

To understand how seawater stimulation induces proinflammatory cytokine production, the effects of NF- $\kappa \mathrm{B}$ and TRPV1 inhibition were compared. As presented in Fig. 5, cells pretreated with either PDTC (NF-кB inhibitor) or, capsazepine (TRPV1 inhibitor) attenuated the release of TNF- $\alpha$ and IL-1 $\beta$ elicited by the seawater challenge. PDTC and capsazepine inhibited the increase in TNF- $\alpha$ and IL- $1 \beta$ concentrations observed following seawater challenge. By contrast, blockage of TRPV4 channels using HC067047 exhibited no effect on the levels of these cytokines (Fig. 5). These results were consistent 

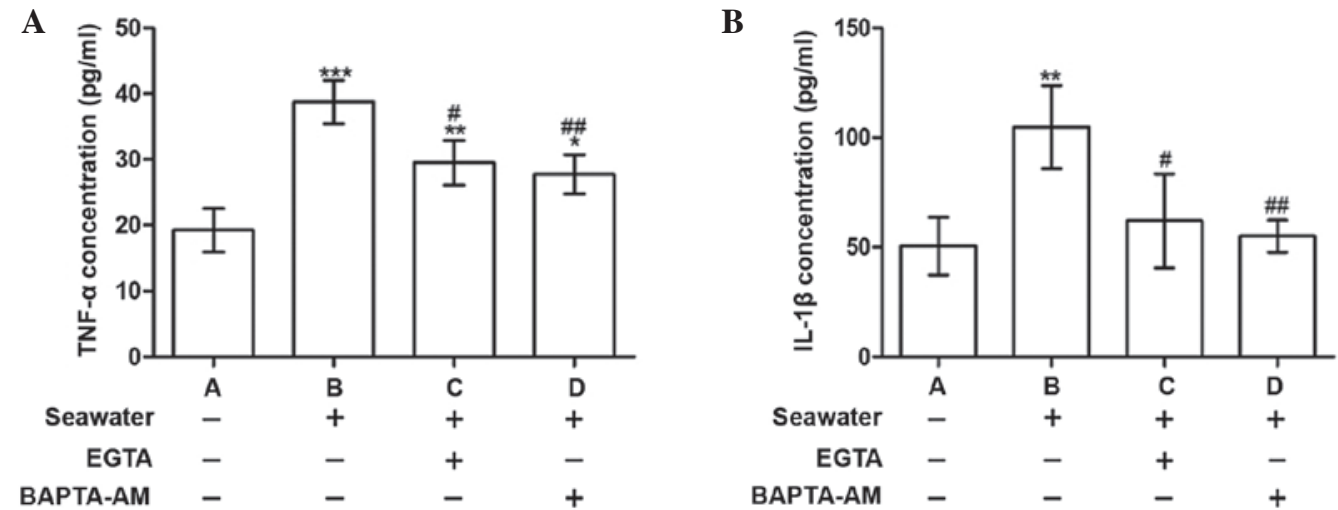

Figure 2. Effect of chelation of calcium on the secretion of TNF- $\alpha$ and IL-1 $\beta$ in A549 cells. (A) TNF- $\alpha$ and (B) IL-1 $\beta$ concentrations following control, seawater, EGTA and BAPTA-AM treatments. ${ }^{*} \mathrm{P}<0.05,{ }^{* *} \mathrm{P}<0.01$ and ${ }^{* * * *} \mathrm{P}<0.001$ vs. the untreated control; ${ }^{*} \mathrm{P}<0.05$ and ${ }^{\# \#} \mathrm{P}<0.01$ vs. seawater treatment. TNF- $\alpha$, tumor necrosis factor $\alpha$; IL-1 $\beta$; interleukin $1 \beta$.

$\mathbf{A}$

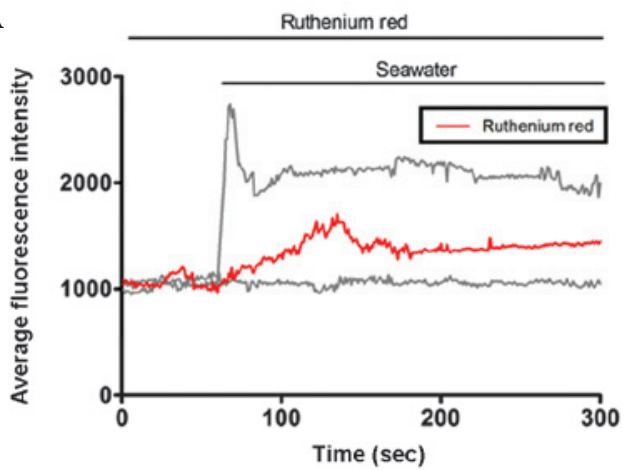

$\mathbf{C}$

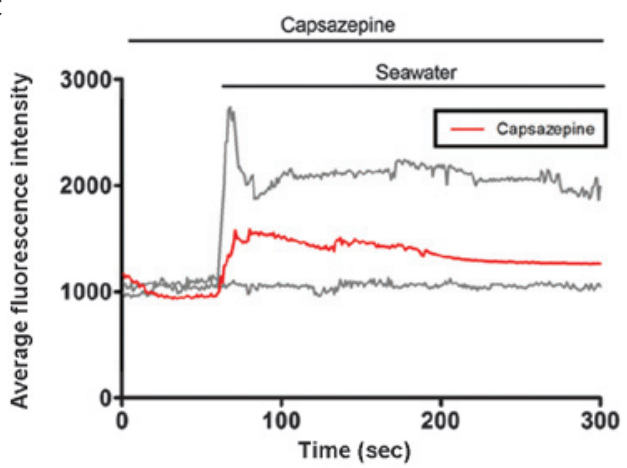

B

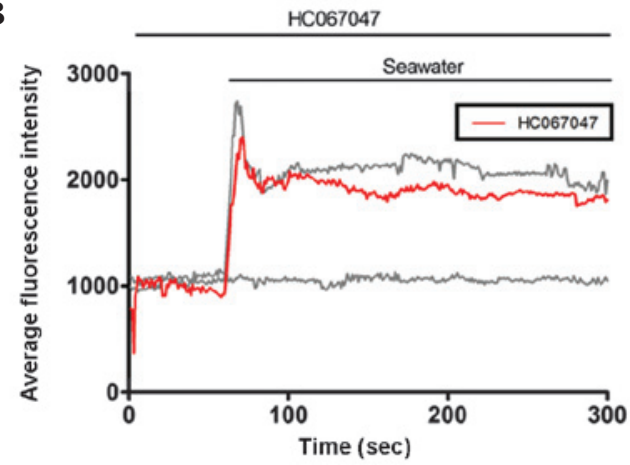

D

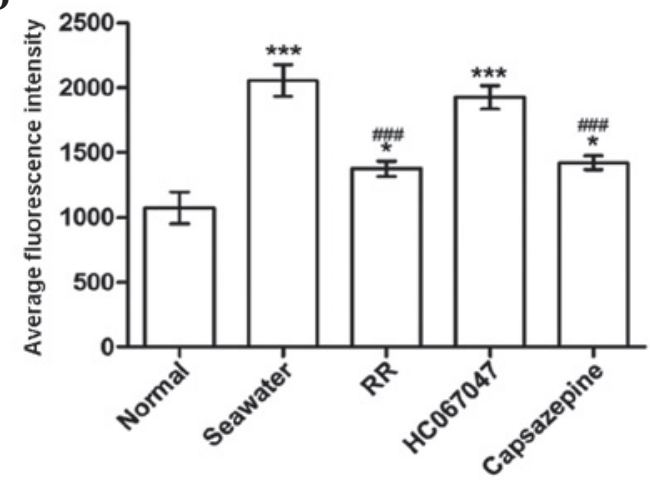

Figure 3. Fluorescence intensity in A549 cells. Effect of (A) RR, (B) HC067047 and (C) capsazepine treatments on average fluorescence intensity in A549 cells. (D) Summary of average fluorescence intensities of each treatment group. ${ }^{*} \mathrm{P}<0.05$ and ${ }^{* * * *} \mathrm{P}<0.001$ vs. the normal group, ${ }^{\# \# \#} \mathrm{P}<0.001$ vs. the seawater group. $\mathrm{RR}$, ruthenium red.

with the finding that seawater challenge evoked extracellular $\mathrm{Ca}^{2+}$ influx by activating TRPV1 channels rather than TRPV4.

Changes in the actin cytoskeleton of A549 cells exposed to seawater were diminished by calcium chelation. To explore the effect of seawater exposure on the actin cytoskeleton, cells were fixed and stained to visualize the actin cytoskeleton using Acti-stain 488 phalloidin. In the control cells, actin filaments were observed to be in a regular arrangement and evenly distributed in the cytoplasm (Fig. 6). By contrast, following seawater challenge, cells exhibited a marked disorganization of actin filaments, formation of stress fibers under the plasma membrane and a dense ring of F-actin was located at the periphery of the cells (Fig. 6). It has previously been reported that cytosolic free $\mathrm{Ca}^{2+}$ oscillation can act as a mediator of actin reorganization (24). To verify whether intracellular calcium oscillation is a prerequisite for the remodeling of F-actin under these conditions, intracellular calcium was chelated by the preincubation of cells with BAPTA-AM or capsazepine. BAPTA-AM partially reversed the disruption of the actin cytoskeleton. However, the TRPV1-specific inhibitor, capsazepine, had no effect on the F-actin distribution (Fig. 6).

Seawater instillation induced lung injury in a $\mathrm{Ca}^{2+}$-dependent manner. The pathology of seawater drowning-induced ALI is characterized by simultaneous neutrophil infiltration, 

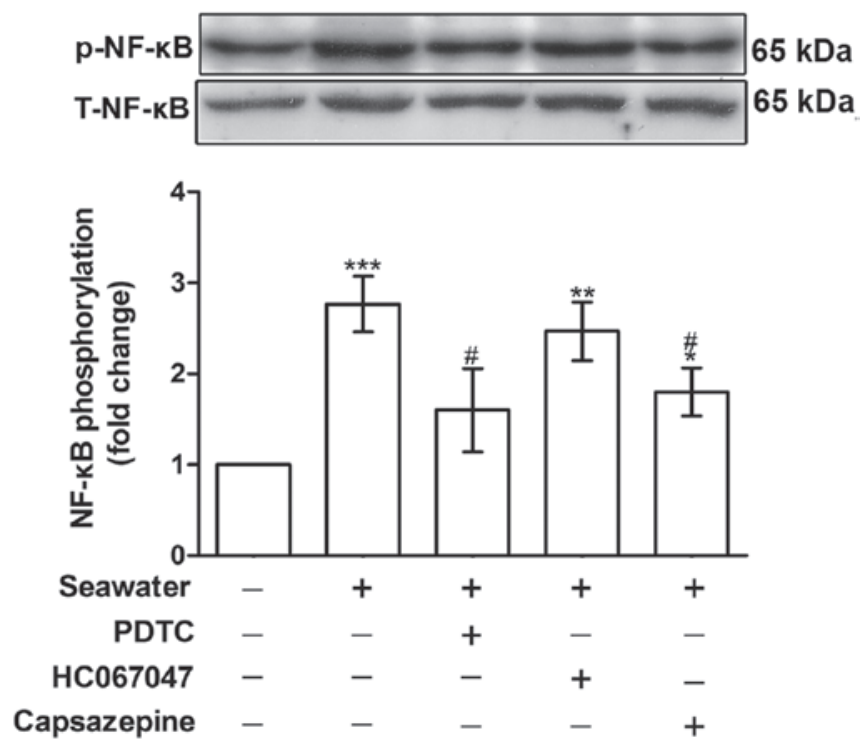

Figure 4. Effect of PDTC, HC067047 and capsazepine treatments on the phosphorylation of NF-kB. ${ }^{*} \mathrm{P}<0.05,{ }^{* *} \mathrm{P}<0.01$ and ${ }^{* * * *} \mathrm{P}<0.001$ vs. the control group, ${ }^{\#} \mathrm{P}<0.05$ vs. the seawater group. PDTC, pyrrolidine dithiocarbamate; NF- $\kappa \mathrm{B}$, nuclear factor $\mathrm{\kappa B}$; $\mathrm{p}$, phospho; $\mathrm{T}$, total.

pulmonary edema with hemorrhage, and production of inflammatory mediators $(25,26)$. Therefore, to assess the severity of lung injury, TNF- $\alpha$ and IL-1 $\beta$ levels were examined, lung MPO activity was measured to determine the levels of neutrophil sequestration and histopathological examination of lung tissues was conducted. These results are presented in Fig. 7. In order to investigate the effect of high concentration of $\mathrm{Ca}^{2+}$ ions in seawater on the severity of lung injury, the $\mathrm{Ca}^{2+}$ in seawater was replaced with $\mathrm{NaCl}$, followed by $\mathrm{pH}$ and osmotic pressure adjustment. Histopathological examination of lung tissues exposed to seawater displayed alveolar collapse, infiltration of inflammatory cells, septal thickening and interstitial edema, demonstrating that seawater challenge induced acute congestion in the lung tissues, in addition to edema and inflammation (Fig. 7A). However, fewer changes to the lung histoarchitecture were observed in the $\mathrm{Ca}^{2+}$-free and isotonic seawater groups (Fig. 7Ac and d).

The seawater group demonstrated significantly increased MPO activity and number of the cells in the BALF, whereas the $\mathrm{Ca}^{2+}$-free seawater and isotonic seawater groups exhibited relatively lower levels compared with the seawater group (Fig. 7B and C). To assess the barrier permeability of the lung, the leak index of Evans blue dye was assessed. The seawater instillation significantly increased the barrier permeability. However, compared with the seawater group, the $\mathrm{Ca}^{2+}$-free group and isotonic groups exhibited significantly reduced barrier permeability $(\mathrm{P}<0.01$; Fig. 7D). Consistent with these changes, seawater instillation resulted in a significant increase of TNF- $\alpha$, IL- $1 \beta$ and IL- 6 concentrations in lung tissues compared with control rats $(\mathrm{P}<0.001$; Fig. 8). Compared with the seawater group, the levels of TNF- $\alpha$ and IL-1 $\beta$ were reduced by instilling either $\mathrm{Ca}^{2+}$-free seawater or isotonic seawater with no change in $\mathrm{Ca}^{2+}$ concentration (Fig. 8).

TRPVI-mediated calcium oscillations connect hypertonia signals and alterations during seawater inhalation-induced

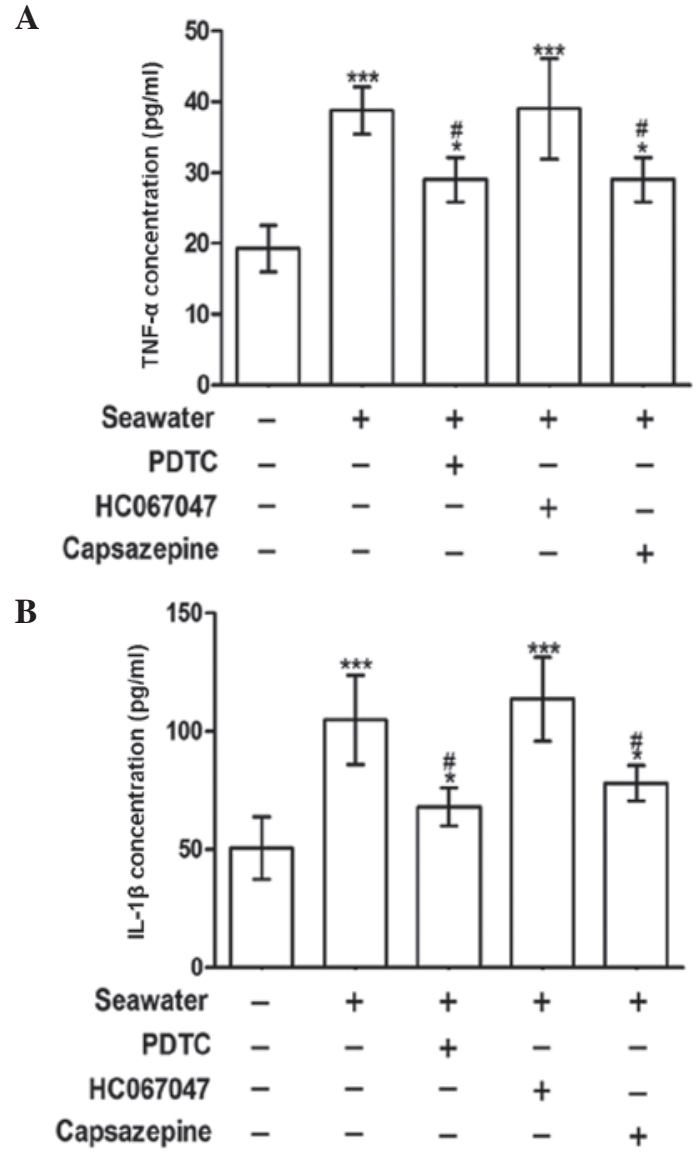

Figure 5. Effect of PDTC, HC067047 and capsazepine treatment on the secretion of TNF- $\alpha$ and IL-1 $\beta$. (A) TNF- $\alpha$ and (B) IL-1 $\beta$ concentrations were measured following control, seawater and seawater + PDTC, HC067047 or capsazepine treatments. ${ }^{*} \mathrm{P}<0.05$ and ${ }^{* * * *} \mathrm{P}<0.001$ vs. the control group, ${ }^{\#} \mathrm{P}<0.05$ vs. the seawater group. PDTC, pyrrolidine dithiocarbamate; TNF- $\alpha$, tumor necrosis factor $\alpha$; IL-1 $\beta$; interleukin $1 \beta$.

lung injury. As summarized in Fig. 9, seawater challenge significantly increased $\mathrm{Ca}^{2+}$ oscillations in lung epithelial cells through the activation of TRPV1. Furthermore, increased $\left[\mathrm{Ca}^{2+}\right] \mathrm{c}$ gives rise to the breakdown of the alveolar-capillary barrier, release of proinflammatory molecules, accumulation of neutrophils and disorganization of actin filaments.

\section{Discussion}

Seawater drowning and associated ALI or respiratory failure remain a notable cause of accidental death $(25,26)$. However, the underlying mechanism remains unclear, and requires further exploration. Similar to lipopolysaccharide (LPS) and cecalligation and puncture-induced lung injury, seawater instillation can also induce excessive release of inflammatory mediators, disturb the integrity of the alveolar septal network and increase blood-air barrier permeability (4). However, seawater exposure directly induces serious pulmonary interstitial edema, alveolar collapse, disturbance of lung blood-air barrier permeability and subsequent infiltration of inflammatory cells, in addition to the activation of the pulmonary inflammatory cascade.

In the current study, the effect of calcium, a component of seawater, on the inflammatory reactions in seawater 

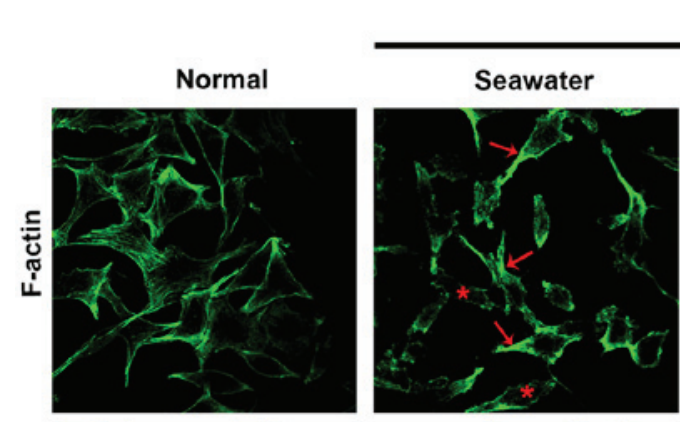

Seawater
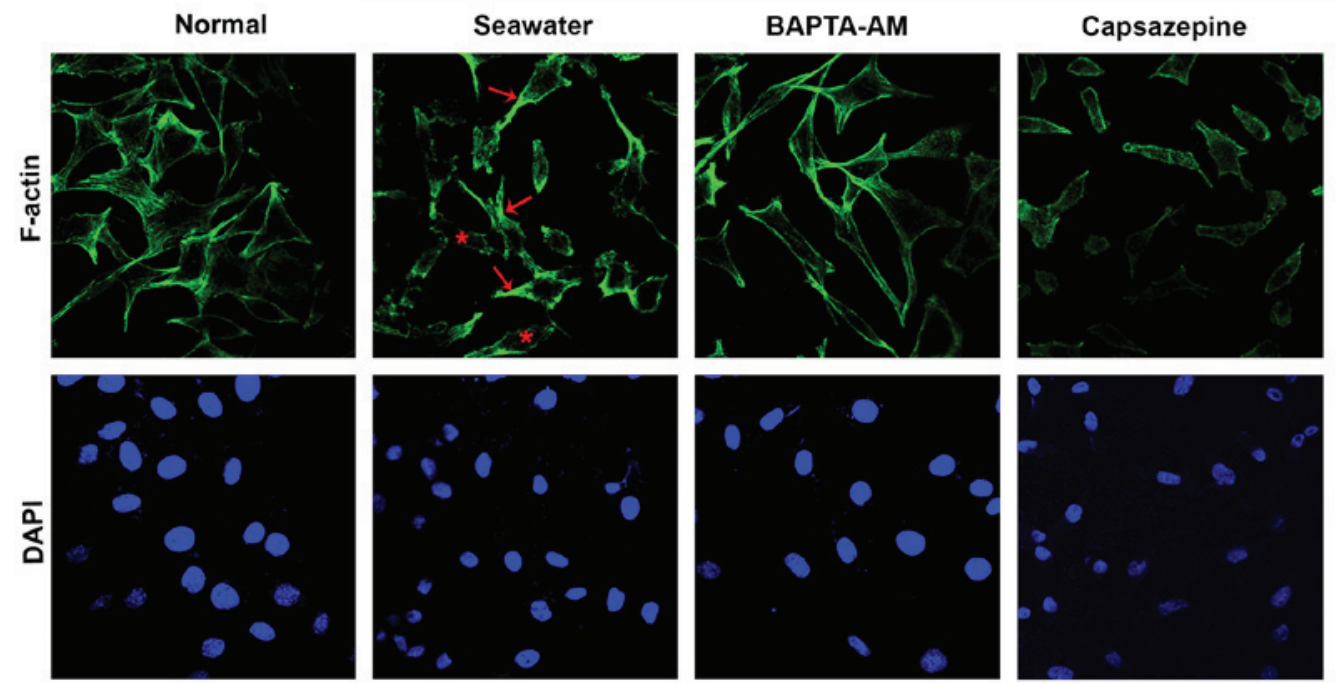

Figure 6. Actin cytoskeleton analysis of A549 cells in the different treatment groups. The actin cytoskeleton was stained with phalloidin-Alexa488, and the nucleus was stained with DAPI, following control, seawater and seawater + BAPTA-AM or capsazepine treatments. Stress fibers are indicated by the red arrows, and the disorganization of actin filaments is indicated by the red asterisks.
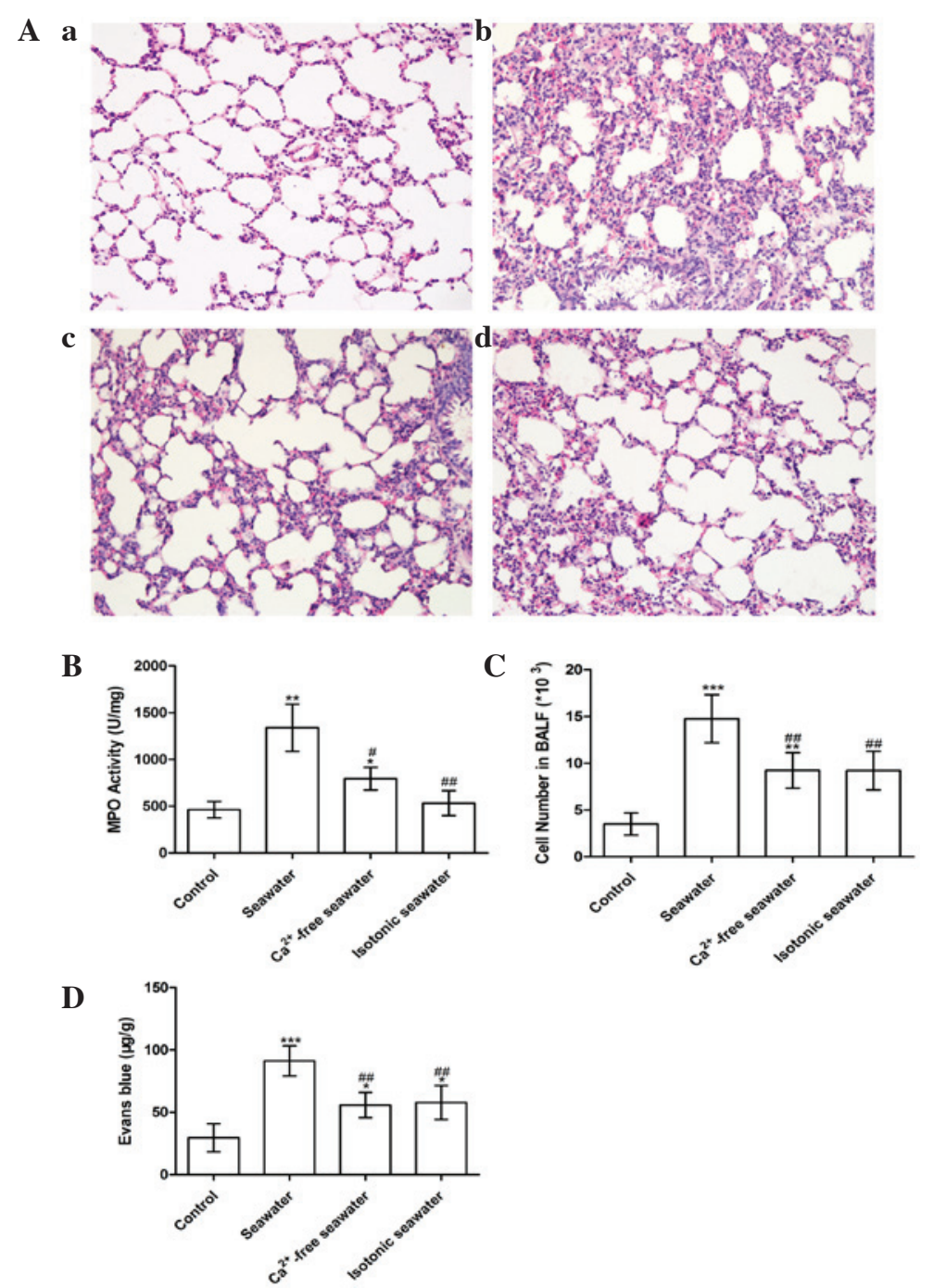

Figure 7. (A) Histopathological changes in lung tissue in the (a) control; (b) seawater; (c) $\mathrm{Ca}^{2+}$-free seawater; and (d) isotonic seawater groups (hematoxylin and eosin staining; magnification, x200). (B) MPO activity of lung tissue; (C) total cells in the BALF; and (D) leak index of Evans blue in the control, seawater, $\mathrm{Ca}^{2+}$-free and isotonic groups. ${ }^{*} \mathrm{P}<0.05,{ }^{* *} \mathrm{P}<0.01$ and ${ }^{* * *} \mathrm{P}<0.001$ vs. the control group, ${ }^{\#} \mathrm{P}<0.05$ and ${ }^{\# \#} \mathrm{P}<0.01$ vs. the seawater group. MPO, myeloperoxidase; BALF, bronchoalveolar lavage fluid. 

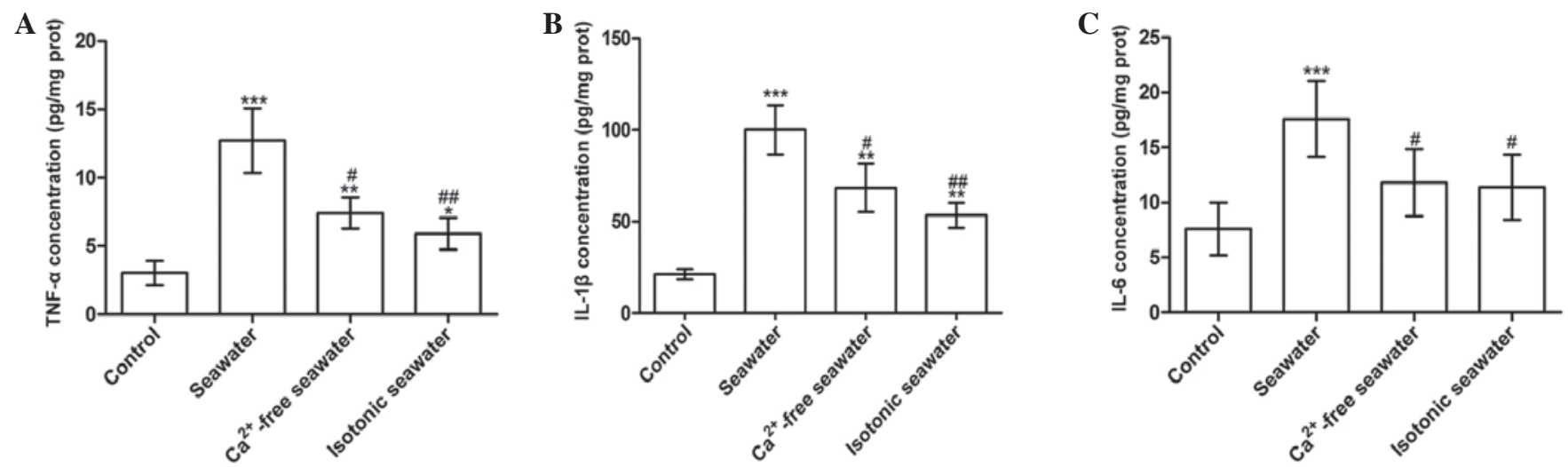

Figure 8. Inflammatory mediators of lung tissue treated with seawater, $\mathrm{Ca}^{2+}$-free and isotonic seawater. (A) TNF- $\alpha$; (B) IL-1 $\beta$; and (C) IL-6 concentrations were measured in the control, seawater, $\mathrm{Ca}^{2+}$-free seawater and isotonic seawater groups. ${ }^{*} \mathrm{P}<0.05,{ }^{* * *} \mathrm{P}<0.01$ and ${ }^{* * * *} \mathrm{P}<0.001$ vs. the control group, ${ }^{*} \mathrm{P}<0.05$ and ${ }^{\#} \mathrm{P}<0.01$ vs. the seawater group. TNF- $\alpha$, tumor necrosis factor $\alpha$; IL; interleukin.

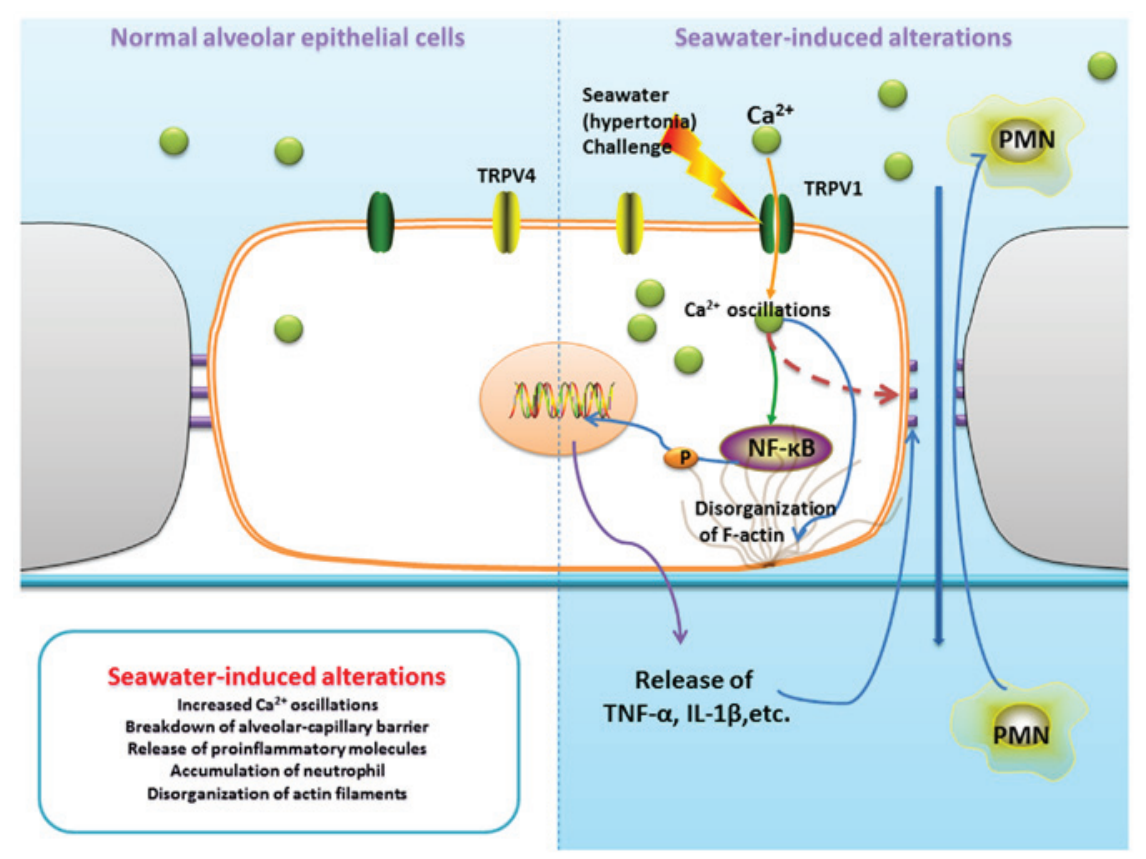

Figure 9. TRPV1-mediated calcium oscillations connect hypertonic signals and alterations during seawater inhalation-induced lung injury. TRPV1, transient receptor potential-vanilloid 1; PMN, polymorphonuclear cells; TNF- $\alpha$, tumor necrosis factor $\alpha$; IL-1 $\beta$; interleukin $1 \beta$.

drowning-induced ALI was investigated. It was demonstrated that high-concentration $\mathrm{Ca}^{2+}$ in seawater exacerbated lung injury. Further study revealed that seawater challenge elevated $\left[\mathrm{Ca}^{2+}\right] \mathrm{c}$ by inducing calcium entry from the extracellular medium via TRPV1 channels. Elevated $\left[\mathrm{Ca}^{2+}\right] \mathrm{c}$ may have induced the increased release of TNF- $\alpha$ and IL-1 $\beta$. It was speculated that these inflammatory reactions were associated with the activation of $\mathrm{NF}-\kappa \mathrm{B}$. Indeed, the study observed that the elevated $\left[\mathrm{Ca}^{2+}\right] \mathrm{c}$ led to greater phosphorylation of NF $-\kappa \mathrm{B}$ (Fig. 4) and increased TNF- $\alpha$ and IL-1 $\beta$ levels. This was corroborated by the diminished inflammatory response following $\mathrm{Ca}^{2+}$ chelation, suggesting an important role for cytosolic $\mathrm{Ca}^{2+}$ in the regulation of lung inflammation.

Calcium is an important second messenger and regulates a variety of cellular functions (6). It is recognized that ALI is often dependent upon cytosolic free $\mathrm{Ca}^{2+}$ oscillation, and that $\mathrm{Ca}^{2+}$ entry into lung endothelium can participate in mediating microvascular-barrier permeability and the inflammatory response to high vascular pressure, hydrogen sulfide or LPS. Alvarez et al (6) reported that disruption of the alveolar septal barrier resulting from $\mathrm{Ca}^{2+}$ influx led to alveolar flooding and impaired gas exchange. Consistent with these findings, Jian et al (8) reported the HiPv-induced increases in $\mathrm{Kf}$ were attenuated by low extracellular $\mathrm{Ca}^{2+}$.

In the present experimental model, seawater challenge resulted in a $\left[\mathrm{Ca}^{2+}\right] \mathrm{c}$ influx characterized by rapid increase to a maximum level within $30 \mathrm{sec}$, followed by a recovery period and sustained plateau. The source of $\mathrm{Ca}^{2+}$ was clarified by chelation of $\mathrm{Ca}^{2+}$ in the extracellular medium using EGTA, which resulted in a weakened increase in $\left[\mathrm{Ca}^{2+}\right] \mathrm{c}$ following exposure to seawater, and the result was confirmed by the re-addition of $\mathrm{Ca}^{2+}$ to the cells. These results indicated that elevation of $\left[\mathrm{Ca}^{2+}\right] \mathrm{c}$ evoked by seawater exposure was mainly accomplished by increase of $\mathrm{Ca}^{2+}$ entry. 
Various membrane transport pathways have been identified as mediators of $\mathrm{Ca}^{2+}$ influx during ALI. Tauseef et al (7) demonstrated that endotoxins induce $\mathrm{Ca}^{2+}$ entry in endothelial cells through the activation of transient receptor potential canonical 6 channels in a Toll-like receptor 4-dependent manner. Alvarez et al (6) implicated TRPV4 in the $\mathrm{Ca}^{2+}$ entry-dependent regulation of endothelial permeability, and the permeability response to the TRPV4 agonist was abolished in lungs from $\mathrm{TRPV4}^{-/}$mice. TRPV1 was also reported to participate in sepsis-evoked ALI (27). Pretreatment with capsazepine markedly attenuated pulmonary COX-2 expression in septic mice (27). To clarify which channels were predominantly responsible for mediating $\mathrm{Ca}^{2+}$ entry and the seawater-induced proinflammatory cytokine production in A549 cells, the present study focused on the role of TRPVs and blocked several potential pathways with the inhibitors ruthenium red, capsazepine and HC067047 (Fig. 3). The results revealed that extracellular $\mathrm{Ca}^{2+}$ influx required the activation of TRPV1 channels following seawater challenge and may be significantly reduced by the TRPV1-specific inhibitor, capsazepine, and the TRPV family inhibitor, ruthenium red.

TRPV1 is a cell membrane-bound $\mathrm{Ca}^{2+}$ channel highly expressed in primary sensory neurons (28) and numerous other cell types, including muscle cells, dendrites and airway epithelial cells $(12,19,28)$. When cells are exposed to cytokines, abnormal $\mathrm{pH}$, osmolality and other irritations, intracellular calcium oscillates by activating TRPV1 $(12,18-20)$ and can initiate endoplasmic reticulum stress and cell death in human bronchial epithelial and alveolar cells (19). In cultured human lung cells, the activation of TRPV1 by various stimuli can also promote calcium-dependent cytokine release and acute respiratory inflammation, with similar results reported in human corneal epithelial cells (29). Additionally, other studies have demonstrated that hypertonic stress increased the levels of IL- 6 and the chemoattractant IL- 8 by eliciting NF- $\kappa$ B activation in a TRPV1-dependent manner (29), and that TRPV1 activation altered F-actin organization through extracellular regulated MAP kinase (ERK1/2) and myosin light chain 2 (MLC2) pathways (30).

In view of the pivotal role of TRPV1 and $\mathrm{Ca}^{2+}$ mobilization in the mediation of inflammation, endoplasmic reticulum stress, cell death and reorganization of the cytoskeleton, TRPV1 was selectively inhibited by capsazepine in vitro to elucidate the function of TRPV1 on seawater drowning-induced ALI. The results demonstrated that seawater exposure gave rise to $N F-\kappa B$ phosphorylation and capsazepine or $\mathrm{Ca}^{2+}$ chelation reduced the effect. Cells pretreated with either capsazepine or an $\mathrm{NF}-\kappa \mathrm{B}$ inhibitor, PDTC, attenuated the increase of TNF- $\alpha$ and IL-1 $\beta$ release elicited by seawater challenge. Thus, seawater challenge may increase the release of proinflammatory cytokines through the phosphorylation and activation of $\mathrm{NF}-\kappa \mathrm{B}$. A549 cells exhibited a marked disorganization of actin filaments and formation of stress fibers following exposure to seawater, whereas changes to the actin cytoskeleton were diminished by pre-incubation of cells with the $\mathrm{Ca}^{2+}$ chelator, BAPTA-AM. However, pretreatment with the TRPV1-specific inhibitor, capsazepine, produced no observed effect on the F-actin distribution. It was surmised that this may be due to capsazepine only being able to diminish calcium influx and ERK1/2 and MLC2 activation, rather than abolish them completely, a slight change to $\mathrm{Ca}^{2+}$ influx may be sufficient to cause F-actin redistribution. Furthermore, seawater may initiate such changes through various other pathways. Further work is necessary to elucidate this mechanism.

In conclusion, these observations place cytosolic $\mathrm{Ca}^{2+}$ ions and TRPV1 at the center of the signaling pathways that mediate seawater drowning-induced ALI, due to their roles in modulating lung inflammation and the cytoskeleton. The present study observed that high-concentration $\mathrm{Ca}^{2+}$ in seawater exacerbated lung injury, and seawater challenge elevated $\left[\mathrm{Ca}^{2+}\right] \mathrm{c}$ by activating TRPV1 channels, potentially leading to the phosphorylation of $\mathrm{NF}-\kappa \mathrm{B}$ and subsequent increased release of TNF- $\alpha$ and IL- $1 \beta$.

\section{Acknowledgements}

The current study was supported by the National Natural Science Foundation of China (grant no. 81270124) and the Military Key Projects in the 12th Five-year Plan of China (project no. CWS13J043).

\section{References}

1. van Beeck EF, Branche CM, Szpilman D, Modell JH and Bierens JJ: A new definition of drowning: Towards documentation and prevention of a global public health problem. Bull World Health Organ 83: 853-856, 2005.

2. Engel SC: Drowning episodes: Prevention and resuscitation tips. J Fam Pract 64: E1-E6, 2015.

3. Salomez F and Vincent JL: Drowning: A review of epidemiology, pathophysiology, treatment and prevention. Resuscitation 63: 261-268, 2004.

4. Ma L, Li Y, Zhao Y, Wang Q, Nan Y, Mu D, Li W, Sun R, Jin F, Liu X: 3,5,4'-tri-O-acetylresveratrol ameliorates seawater exposure-induced lung injury by upregulating connexin 43 expression in lung. Mediators Inflamm 2013: 182132, 2013.

5. Li J, Xu M, Fan Q, Xie X, Zhang Y, Mu D, Zhao P, Zhang B, Cao F, Wang Y, et al: Tanshinone IIA ameliorates seawater exposure-induced lung injury by inhibiting aquaporins (AQP) 1 and AQP5 expression in lung. Respir Physiol Neurobiol 176: 39-49, 2011.

6. Alvarez DF, King JA, Weber D, Addison E, Liedtke W and Townsley MI: Transient receptor potential vanilloid 4-mediated disruption of the alveolar septal barrier: A novel mechanism of acute lung injury. Circ Res 99: 988-995, 2006.

7. Tauseef M, Knezevic N, Chava KR, Smith M, Sukriti S, Gianaris N, Obukhov AG, Vogel SM, Schraufnagel DE, Dietrich A, et al: TLR4 activation of TRPC6-dependent calcium signaling mediates endotoxin-induced lung vascular permeability and inflammation. J Exp Med 209: 1953-1968, 2012.

8. Jian MY, King JA, Al-Mehdi AB, Liedtke W and Townsley MI: High vascular pressure-induced lung injury requires $\mathrm{P} 450$ epoxygenase-dependent activation of TRPV4. Am J Respir Cell Mol Biol 38: 386-392, 2008.

9. Pan Z, Wang Z, Yang H, Zhang F and Reinach PS: TRPV1 activation is required for hypertonicity-stimulated inflammatory cytokine release in human corneal epithelial cells. Invest Ophthalmol Vis Sci 52: 485-493, 2011.

10. Caterina MJ, Schumacher MA, Tominaga M, Rosen TA, Levine JD and Julius D: The capsaicin receptor: a heat-activated ion channel in the pain pathway. Nature 389: 816-824, 1997.

11. Nishihara E, Hiyama TY and Noda M: Osmosensitivity of transient receptor potential vanilloid 1 is synergistically enhanced by distinct activating stimuli such as temperature and protons. PLoS One 6: e22246, 2011.

12. Geppetti P, Materazzi S and Nicoletti P: The transient receptor potential vanilloid 1: Role in airway inflammation and disease. Eur J Pharmacol 533: 207-214, 2006.

13. Sadofsky LR, Ramachandran R, Crow C, Cowen M, Compton SJ and Morice AH: Inflammatory stimuli up-regulate transient receptor potential vanilloid-1 expression in human bronchial fibroblasts. Exp Lung Res 38: 75-81, 2012. 
14. Thomas KC, Robers, JK, Deering-Rice CE, Romero EG, Dull RO, Lee J, Yost GS and Reilly CA: Contributions of TRPV1, endovanilloids, and endoplasmic reticulum stress in lung cell death in vitro and lung injury. Am J Physiol Lung Cell Mol Physiol 302: L111-L119, 2012.

15. Hamanaka K, Jian MY, Weber DS, Alvarez DF, Townsley MI, Al-Mehdi AB, King JA, Liedtke W and Parker JC: TRPV4 initiates the acute calcium-dependent permeability increase during ventilator-induced lung injury in isolated mouse lungs. Am J Physiol Lung Cell Mol Physiol 293: L923-L932, 2007.

16. Liu L, Chen L, Liedtke W and Simon SA: Changes in osmolality sensitize the response to capsaicin in trigeminal sensory neurons. J Neurophysiol 97: 2001-2015, 2007.

17. Sidhaye VK, Guler AD, Schweitzer KS, D'Alessio F, Caterina MJ and King LS: Transient receptor potential vanilloid 4 regulates aquaporin-5 abundance under hypotonic conditions. Proc Nat Acad Sci USA 103: 4747-4752, 2006.

18. Johansen ME, Reilly CA and Yost GS: TRPV1 antagonists elevate cell surface populations of receptor protein and exacerbate TRPV1-mediated toxicities in human lung epithelial cells Toxicol Sci 89: 278-286, 2006.

19. Thomas KC, Sabnis AS, Johansen ME, Lanza DL, Moos PJ, Yost GS and Reilly CA: Transient receptor potential vanilloid 1 agonists cause endoplasmic reticulum stress and cell death in human lung cells. J Pharmacol Exp Ther 321: 830-838, 2007.

20. Lee LY and Gu Q: Role of TRPV1 in inflammation-induced airway hypersensitivity. Curr Opin Pharmacol 9: 243-249, 2009.

21. Guler AD, Lee H, Iida T, Shimizu I, Tominaga M and Caterina M: Heat-evoked activation of the ion channel, TRPV4. J Neurosci 22: 6408-6414, 2002.

22. Liedtke W, Choe Y, Marti-Renom MA, Bell AM, Denis CS, Sali A, Hudspeth AJ, Friedman JM and Heller S: Vanilloid receptor-related osmotically activated channel (VR-OAC), a candidate vertebrate osmoreceptor. Cell 103: 525-535, 2000.
23. O'Neil RG and Heller S: The mechanosensitive nature of TRPV channels. Pflugers Arch 451: 193-203, 2005.

24. Rosado JA, González A, Salido GM and Pariente JA: Effects of reactive oxygen species on actin filament polymerisation and amylase secretion in mouse pancreatic acinar cells. Cell Signal 14: 547-556, 2002.

25. Ma L, Zhao Y, Li B, Wang Q, Liu X, Chen X, Nan Y, Liang L, Chang R, Liang L, et al: 3,5,4'-Tri-O-acetylresveratrol attenuates seawater aspiration-induced lung injury by inhibiting activation of nuclear factor-kappa $B$ and hypoxia-inducible factor-1 $\alpha$. Respir Physiol Neurobiol 185: 608-614, 2013.

26. Fan Q, Zhao P, Li J, Xie X, Xu M, Zhang Y, Mu D, Li W, et al: $17 \beta$-Estradiol administration attenuates seawater aspiration-induced acute lung injury in rats. Pulm Pharmacol The 24: 673-681, 2011.

27. Ang SF, Sio SW, Moochhala SM, MacAry PA and Bhatia M: Hydrogen sulfide upregulates cyclooxygenase- 2 and prostaglandin E metabolite in sepsis-evoked acute lung injury via transient receptor potential vanilloid type 1 channel activation. J Immunol 187: 4778-4787, 2011.

28. Cortright DN and Szallasi A: Biochemical pharmacology of the vanilloid receptor TRPV1. An update. Eur J Biochem 271: 1814-1819, 2004.

29. Reilly CA, Taylor JL, Lanza DL, Carr BA, Crouch DJ and Yost GS: Capsaicinoids cause inflammation and epithelial cell death through activation ofvanilloid receptors. Toxicol Sci 73: 170-181, 2003.

30. Cong X, Zhang Y, Yang NY, Li J, Ding C, Ding QW, Su QC, Mei M, Guo XH, Wu LL and Yu GY: Occludin is required for TRPV1-modulated paracellular permeability in the submandibular gland. J Cell Sci 26: 1109-1121, 2013. 